Article

\title{
Predictive Risk of Radiation Induced Cerebral Necrosis in Pediatric Brain Cancer Patients after VMAT Versus Proton Therapy
}

\section{Derek Freund ${ }^{1,2, \dagger}$, Rui Zhang ${ }^{1,2, *}$, Mary Sanders ${ }^{1}$ and Wayne Newhauser ${ }^{1,2}$}

1 Department of Radiation Oncology, Mary Bird Perkins Cancer Center, 4950 Essen Ln., Baton Rouge, LA 70809, USA; E-Mails: dfreund@wkhs.com (D.F.); msanders@marybird.com (M.S.); wnewhauser@marybird.com (W.N.)

2 Department of Physics and Astronomy, Louisiana State University, Nicholson Hall, Tower Dr., Baton Rouge, LA 70810, USA

$\dagger$ Current address: Department of Radiation Oncology, Willis Knighton Cancer Center, 2600 Kings Hwy, Shreveport, LA 71103, USA.

* Author to whom correspondence should be addressed; E-Mail: rzhang@marybird.com.

Academic Editor: Xiaodong Zhang

Received: 1 January 2015 / Accepted: 25 March 2015 / Published: 10 April 2015

\begin{abstract}
Cancer of the brain and central nervous system(CNS) is the second most common of all pediatric cancers. Treatment of many of these cancers includes radiation therapy of which radiation induced cerebral necrosis (RICN) can be a severe and potentially devastating side effect. Risk factors for RICN include brain volume irradiated, the dose given per fraction and total dose. Thirteen pediatric patients were selected for this study to determine the difference in predicted risk of RICN when treating with volumetric modulated arc therapy (VMAT) compared to passively scattered proton therapy (PSPT) and intensity modulated proton therapy (IMPT). Plans were compared on the basis of dosimetric endpoints in the planned treatment volume (PTV) and brain and a radiobiological endpoint of RICN calculated using the Lyman-Kutcher-Burman probit model. Uncertainty tests were performed to determine if the predicted risk of necrosis was sensitive to positional errors, proton range errors and selection of risk models. Both PSPT and IMPT plans resulted in a significant increase in the maximum dose to the brain, a significant reduction in the total brain volume irradiated to low doses, and a significant lower predicted risk of necrosis compared with the VMAT plans. The findings of this study were upheld by the uncertainty analysis.
\end{abstract}


Keywords: radiation induced cerebral necrosis; brain cancer; volumetric modulated arc therapy; proton therapy

\section{Introduction}

Cancer of the brain and central nervous system (CNS) is the second most common of all pediatric cancers. At a rate of 5.1 out of every 100,000 cases per year, its incidence is second only to leukemia [1]. The Surveillance Epidemiology and End Result (SEER) program reported that astrocytomas and other gliomas make up approximately $67 \%$ of pediatric brain cancers, and ependymomas make up $9 \%$ [2]. Treatment for these tumors often incorporates radiation therapy and/or chemotherapy [3] with typical prescriptions of 50-60 Gy in 1.8-2Gy/fraction to the planned treatment volume (PTV) [4]. Proton therapies have been administered up to 59.4 Gy (RBE) to the PTV which includes the gross tumor volume (GTV) plus 2-5 cm expansion [5].

The potential side effects from CNS irradiation can include acute effects and late effects. Acute affects are seen early and can include alopecia, erythema, otitis, tinnitus, or even temporary demyelination [3]. Alternatively late effects onset more than 6 months after treatment and tend to be more severe, including spinal myelopathy, endocrine and cognitive dysfunction, secondary cancers, and radiation induced necrosis [6]. With approximately $72 \%$ and $73 \%$ five-year survival rate of patients with primary brain and CNS tumors between the ages 0-14 and 0-19, respectively, the risk of late effects is a major concern [7].

Radiation induced cerebral necrosis (RICN) has a latency of as little as 3 month or as long as 13 year after treatment and can vary in severity from asymptomatic radiographic changes, cognitive dysfunction, stroke to death. [8]. Due in part to a long latency, difficulty of diagnosis, and apparently low incidence, the true incidence of RICN is poorly known [9]. Some reports indicate that depending on the treatment protocol the actual incidence could be anywhere from 3\% to 24\% [10]. Although the expected incidence of RICN may be low, it is a severe and potentially lethal late effect that can extremely impact these patients quality of life. Little is known about the mechanism by which RICN occurs but it has been suggested by Fink et al. [8] that it may be a result of ischemia resulting from vascular endothelial injury or from the loss of or injury to oligodendrocytes. Other potential risk factors include the total dose given, the total volume irradiated, fraction size, concurrent chemotherapy, age at exposure, treatment modality, etc. [8,11-14].

Recent retrospective studies have assessed some of these risk parameters. A recent study on the quantitative analysis of normal tissue effects in the clinic (QUANTEC) looked at the radiation dose-volume effects in the brain [12]. Their result indicates that the dose to cause radiation is high with a predicted 5\% and $10 \%$ risk of symptomatic necrosis to occur at 72 Gy and $90 \mathrm{~Gy}$ given in $2 \mathrm{~Gy} /$ fraction increments [12]. Additionally they added that fractions sizes greater than 2 Gy increased the risk. Another recent study by Murphy et al. [14] found that the percentage of the infratentorial brain receiving 50, 52 and 54 Gy were significant predictors of risk of necrosis for pediatric patients being treated for craniopharyngioma. Ruben et al. [10] found that the risk of necrosis increases with increasing dose, fraction size and the addition of chemotherapy.

All of the above studies were based on patients treated with photon therapy. Boehling et al. [15] compared intensity modulated photon therapy (IMRT) with intensity modulated proton therapy (IMPT) 
for pediatrics with craniopharyngioma and found IMPT spared dose to the cerebral vasculature compared to IMRT. Other studies have found similar effects of normal tissue sparing for pediatric medulloblastoma when comparing passively scattered proton therapy (PSPT) to conventional photon therapy [16].

The primary focus of this investigation is to compare the risk of RICN between volumetric modulated arc therapy (VMAT) and passively scattered proton therapy (PSPT) as well as intensity modulated proton therapy (IMPT). Comprehensive uncertainty analysis was also performed.

\section{Methods and Materials}

A patient database was constructed with 13 anonymized CT data sets from pediatric patients with varying age, sex and treatment sites. These patients were consecutively sampled from patients that were previously treated with proton cranial spinal irradiation (CSI) at the University of Texas at M.D. Anderson between 2007 and 2009. Five of these patients were used for our ependymoma comparison and eight for the astrocytoma (glioma) comparison. A list of these patients based on age, sex and disease type can be seen Table 1 .

Contouring was performed in Phillips Pinnacle treatment planning system (TPS). A clinical treatment volume (CTV) was created for each patient by the same board certified radiation oncologist to represent the glioma and ependymoma gross tumor volume (GTV) plus any subclinical disease that may exist. An additional $1 \mathrm{~cm}$ margin was then added to the CTV to define the planned treatment volume (PTV). This additional PTV margin accounts for patient setup error. A PTV reduction of no more than $0.5 \mathrm{~cm}$ was allowed to account for organs at risk or boney anatomy. Additional beam specific proximal margins $(\mathrm{PM})$ and distal margins (DM) were determined for proton plans due to range uncertainties inherent in proton beam therapy [17].

Table 1. Patient index, age, sex, diagnosis used for the study and target volume.

\begin{tabular}{ccccc}
\hline Patient Index & Age at Treatment & Sex & Assigned Diagnosis * & PTV Volume $\left(\mathbf{c m}^{\mathbf{3}}\right)$ \\
\hline 1 & 2 & $\mathrm{~F}$ & Glioma & 89.1 \\
2 & 4 & $\mathrm{M}$ & Glioma & 86.31 \\
3 & 6 & $\mathrm{~F}$ & Glioma & 38.34 \\
4 & 8 & $\mathrm{~F}$ & Glioma & 180.2 \\
5 & 10 & $\mathrm{~F}$ & Glioma & 139.87 \\
6 & 4 & $\mathrm{M}$ & Glioma & 71.4 \\
7 & 6 & $\mathrm{M}$ & Glioma & 76.74 \\
8 & 8 & $\mathrm{M}$ & Glioma & 215.69 \\
9 & 10 & $\mathrm{M}$ & Ependymoma & 64.38 \\
10 & 12 & $\mathrm{~F}$ & Ependymoma & 97.41 \\
11 & 13 & $\mathrm{~F}$ & Ependymoma & 105.75 \\
12 & 16 & $\mathrm{~F}$ & Ependymoma & 72.57 \\
13 & 16 & $\mathrm{~F}$ & Ependymoma & 95.49 \\
\hline
\end{tabular}

* Note: Diagnoses were assigned for the purposes of this study and were different from the diagnoses listed in the medical records.

Volumetric Modulated Arc Therapy (VMAT) plans were constructed in Phillips' Pinnacle TPS, while passively scattered and intensity modulate proton plans were constructed in Varian's Eclipse TPS. A prescription dose of $54 \mathrm{~Gy}(\mathrm{RBE})$ in $1.8 \mathrm{~Gy}(\mathrm{RBE}) /$ fraction over 30 fractions was used for all patients. 
Proton RBE of 1.1 was used in this study according to ICRU recommendation [18]. Variation of proton RBE was not considered in this study for simplicity, although there are concerns about the correlation between necrosis and the uncertainties of proton RBE [19]. Plans were considered provisionally acceptable when the dose to 95\% of the PTV (D95\%) was 95\% (51.3 Gy (RBE)) of the prescription for glioma plans and 90\% (48.6 Gy (RBE)) for ependymoma plans. These dose objectives were maintained unless the constraint for the optic chiasm (50 Gy (RBE)) or brainstem (54 Gy (RBE)) was not met. All plans received final approval by a radiation oncologist.

A post planning comparison was performed to assess the congruence between the photon and proton plans. The plan conformity was evaluated in the PTV using the conformity index (CI) as described by Feuvret et al. [20]

$$
C I=\frac{T V_{R I}}{T V} \times \frac{T V_{R I}}{V_{R I}}
$$

where TV is the target volume (PTV), TVRI is the target volume that is covered by the reference isodose, and $V_{R I}$ is the volume of the reference isodose. In this work the reference isodose was 95\% (51.3 Gy (RBE)) of the prescription dose. Conformity index has a range from 0 to 1 , where 1 is ideal. The Dose to $95 \%$ of the PTV was also used to quantify the coverage of the PTV and can be extracted from the cumulative dose volume histograms (DVH) in tabular form.

The dose homogeneity index is a measure of how homogeneous the dose is within the PTV. This metrics was described by Yoon et al. [21] and is given by

$$
H I=\frac{D_{2 \%}-D_{98 \%}}{D_{p}}
$$

where $\mathrm{D}_{2} \%$ is the dose to $2 \%$ of the PTV volume, $\mathrm{D}_{98 \%} \%$ is the dose to $98 \%$ of the PTV volume, and $\mathrm{D}_{\mathrm{p}}$ is the prescribed dose. Homogeneity index has an optimal value of 0 .

Plans were evaluated on RICN in the brain using the Lyman Kutcher Burman (LKB) probit model for normal tissue complication probability (NTCP) [22]. The ratio of NTCP (rNTCP) was then determined to compare between proton and VMAT plans using the following equation.

$$
r N T C P=\frac{N T C P_{\text {proton }}}{N T C P_{V M A T}}
$$

where the NTCP calculated for protons plans was divided by the NTCP calculated for the VMAT plans.

A sensitivity analysis was used to determine the robustness of treatment plans for comparison. Setup and range uncertainty was simulated using $\pm 1 \mathrm{~cm}$ isocenter shifts and $\pm 10 \%$ Hounsfield to stopping power calibration curve errors respectively. Additionally sensitivity of the risk model to dose and volume parameter may result in changes in the predicted risk of necrosis. For this reason the predicted risk was computed using a variety of the other risk models and compared to the Lyman Kutcher Burman (LKB) model. These other models included the linear no-threshold model (LNT), linear threshold model (LT), linear plateau model (LP) and linear quadratic model (LQ) and were fit to the same total whole brain dose of 60 Gy (RBE) to induce 50\% complication (TD50) as the LKB model [22]. The resulting fit can be seen in Figure 1. 


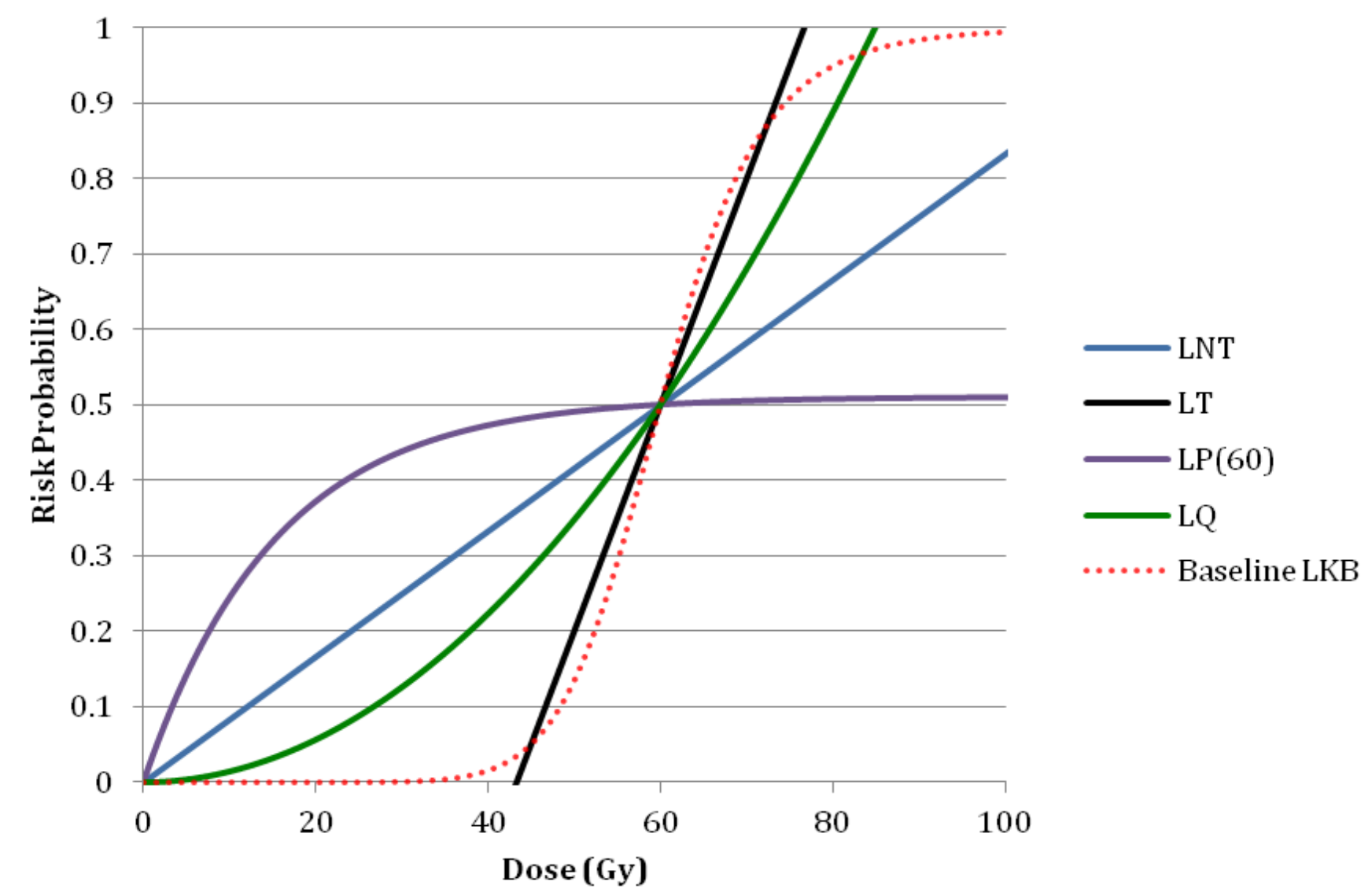

Figure 1. Alternative risk models used to test the sensitivity of rNTCP to the shape of the model used. LNT is the linear no-threshold model, LT is the linear threshold model, LP(60) is the linear plateau model, and LQ is the linear quadratic model.

\section{Results and Discussion}

\subsection{Planning Comparison}

Patient plans were compared on the basis of different dose metrics for the PTV and whole brain. In the PTV, CI, HI and the dose to 95\% of the PTV (D95\%) were used for comparison. Patient isodose distributions and DVH were also used to visually compare patient plan metrics and they are shown in Figures 2 and 3 for a typical patient. The results for CI and HI are displayed in Figure 4a,b respectively.

PSPT plans showed no significant difference in the conformity compared to VMAT with average CI values of $0.58 \pm 0.07$ and $0.62 \pm 0.08$, respectively. The IMPT plans resulted in significantly better conformity than the VMAT plans with an average CI value of $0.83 \pm 0.06$. Merchant et al. [23] also found better conformity over photon therapy when using an active scanning proton technique for a variety of different brain cancer treatments. However the specific photon modality that was used for treatment was not specified in that study. Baumert et al. [24] also found increase conformity for IMPT and PSPT for irregularly shaped tumors compared to intensity modulated stereotactic radiotherapy but conformity was comparable between modalities for large concentric treatment volumes.

Both the VMAT and IMPT plans showed good homogeneity with average HI values of $0.07 \pm 0.05$ and $0.12 \pm 0.12$. On the other hand, PSPT plans had significantly worse homogeneity than the VMAT plans with an average HI of $0.23 \pm 0.19$. Contrary to our study, Bolsi et al. [25] found that PSPT had 
better homogeneity than photons. However their comparison was with 3D conformal radiation therapy and not with VMAT, which typically has better conformity. Howell et al. [16] also found that photon plans had greater dose heterogeneity than passively scattered proton therapy (PSPT) for medulloblastoma. Again, their study was not comparing PSPT to VMAT and additionally they used D5\% and D95\% for homogeneity calculation while $\mathrm{D}_{2 \%}$ and $\mathrm{D}_{98 \%}$ were used in our study. Kozak et al. [26] did compare protons to intensity modulated radiation therapy (IMRT), which has a similar dose delivery to VMAT and found that homogeneity was comparable between modalities.
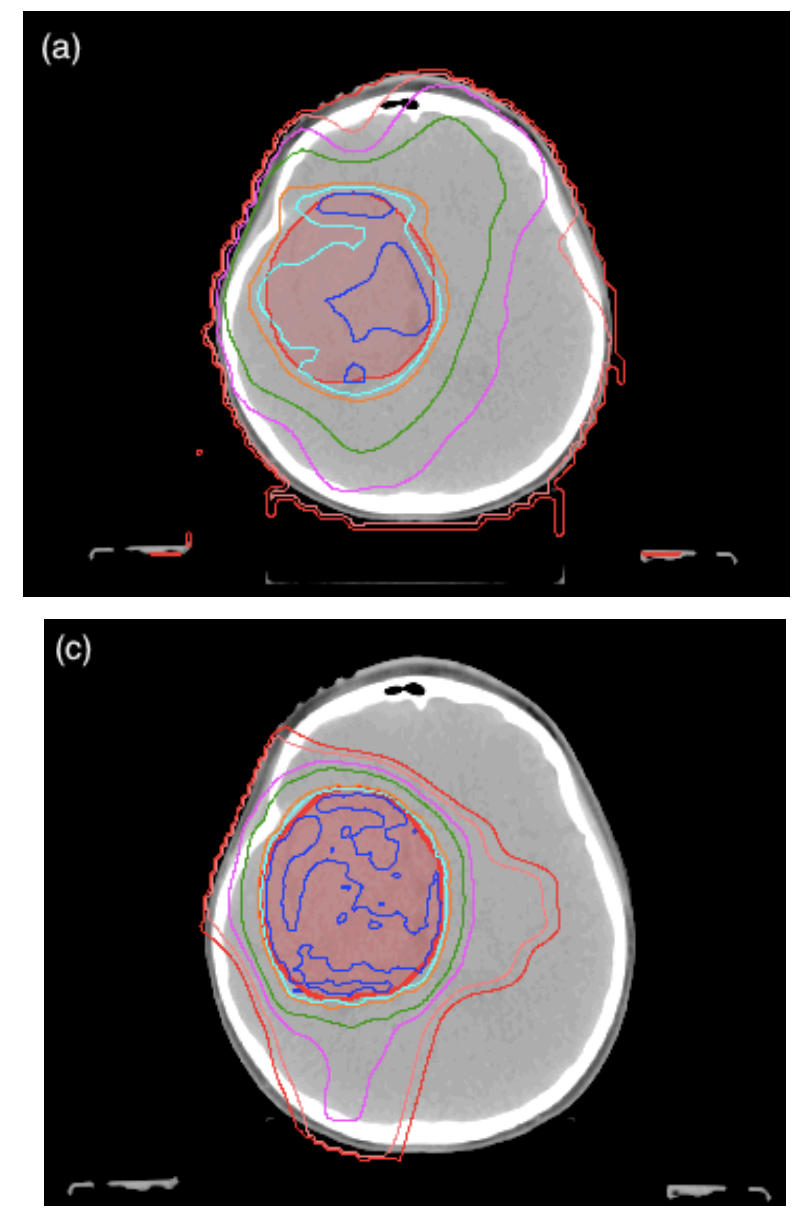

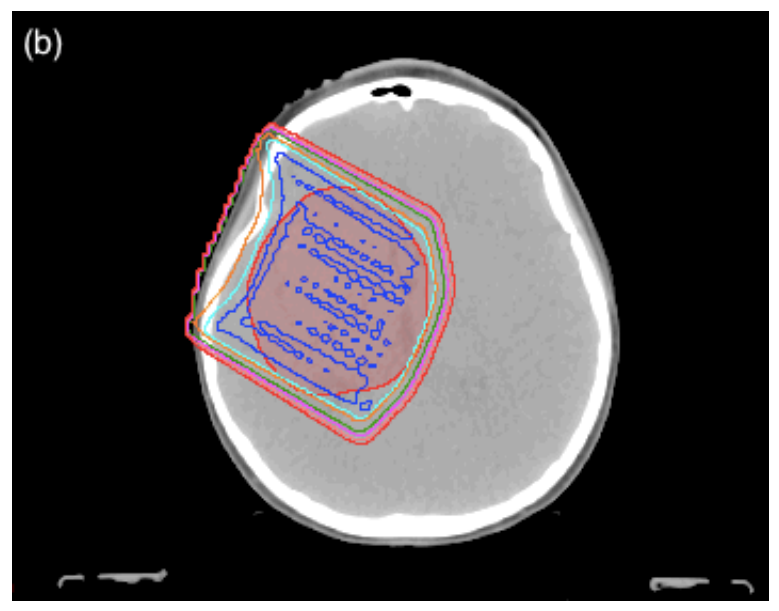

\begin{tabular}{|l||l|}
\hline Isodose & Color \\
\hline \hline $59.4 \mathrm{~Gy}-\mathrm{RBE}$ & \\
\hline $56.7 \mathrm{~Gy}-\mathrm{RBE}$ & \\
\hline $54 \mathrm{~Gy}-\mathrm{RBE}$ & \\
\hline $51.3 \mathrm{~Gy}-\mathrm{RBE}$ & \\
\hline $48.6 \mathrm{~Gy}-\mathrm{RBE}$ & \\
\hline $30 \mathrm{~Gy}-\mathrm{RBE}$ & \\
\hline $20 \mathrm{~Gy}-\mathrm{RBE}$ & \\
\hline $10 \mathrm{~Gy}-\mathrm{RBE}$ & \\
\hline $5 \mathrm{~Gy}-\mathrm{RBE}$ & \\
\hline \hline ROI & Color \\
\hline \hline PTV & colorwash \\
\hline
\end{tabular}

Figure 2. Patient 5 isodose distribution at isocenter CT slice location for (a) volumetric modulated arc therapy (VMAT), (b) passively scattered proton therapy (PSPT), and (c) intensity modulated proton therapy (IMPT).

PSPT plans resulted in a significantly lower average D95\% than the VMAT plans and 5 of the 13 plans did not meet the criteria for acceptable coverage. IMPT and VMAT average D95\% values were not significantly different from one another and only one patient from each modality failed to meet the coverage criteria (patients 10 and 5, respectively). It is important to note that although some of these plans failed to meet the planning criteria they were still considered clinically acceptable. The failure may have been a result of the proximity of the treatment volume with respect to the brainstem. For this reason the PTV planning goal was sacrificed in these cases since the constraint on the dose to the brainstem was considered as the paramount objective. Other studies have also found similar coverage between proton and 
IMRT photon plans but superior coverage for protons when compared against photon conformal radiation therapy $[23,25,27]$.

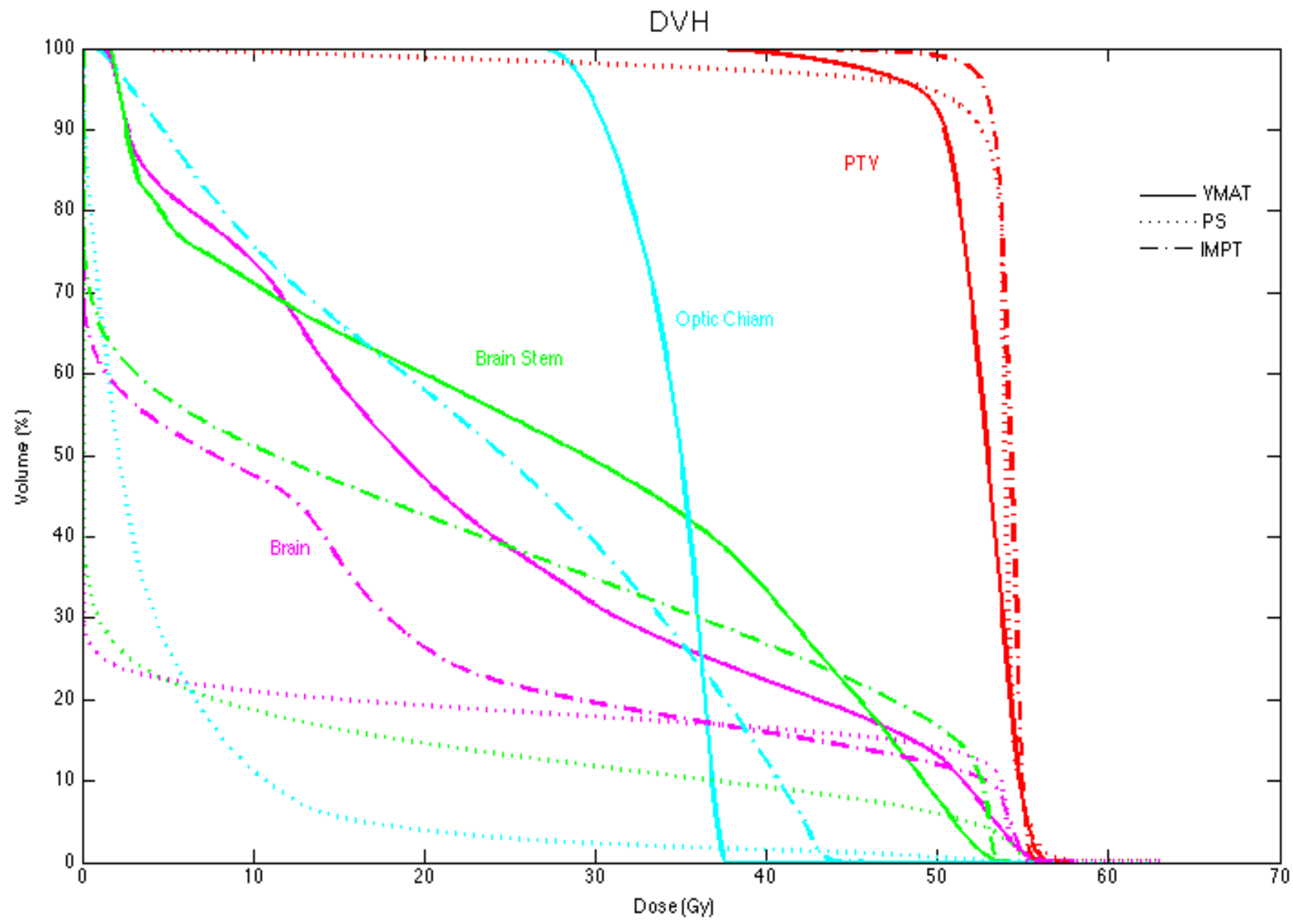

Figure 3. The dose volume histograms (DVH) for patient 5. VMAT plans are represented with the solid lines, PSPT with the dotted lines and IMPT with the dash-dot lines. Different regions of interest are represented with different colored lines.
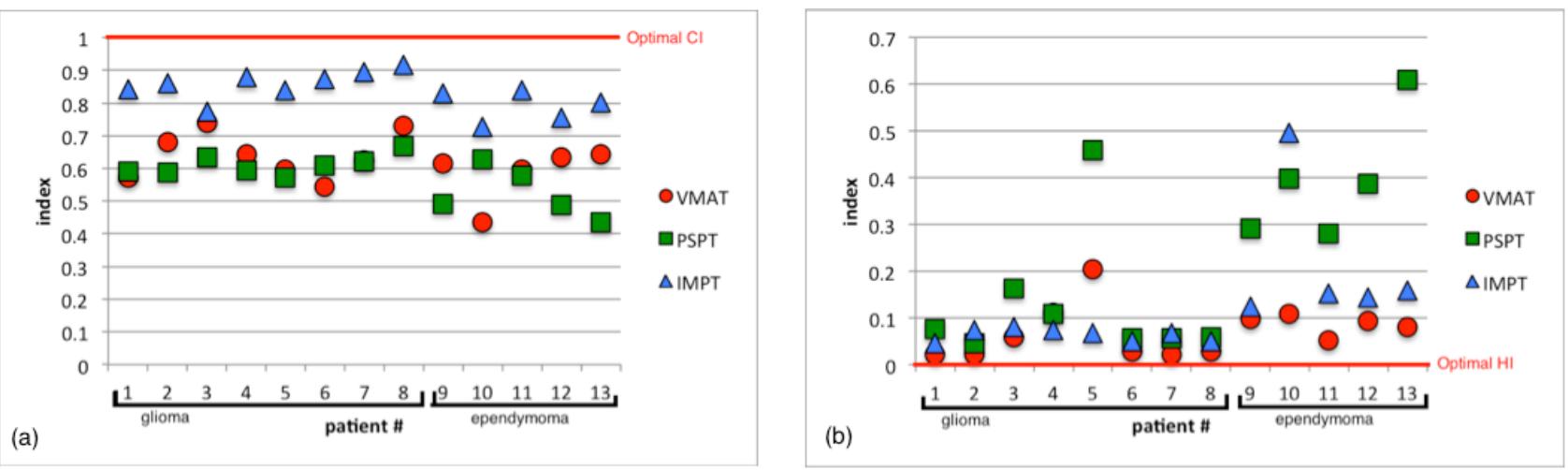

Figure 4. (a) The conformity of the dose to the PTV represented by the conformity index (CI) for VMAT, PSPT, and IMPT plans. (b) The homogeneity of the dose to the PTV represented by the homogeneity index (HI) for VMAT, PSPT, and IMPT plans. 
The whole brain was compared on the basis of maximum dose, mean dose and volume of the brain receiving 5, 10, 50, 52, and $56 \mathrm{~Gy}(\mathrm{RBE})$. The maximum dose to the brain for all patients is displayed in Figure 5. Both IMPT and PSPT plans resulted in a significantly higher maximum dose to the brain than the VMAT plans with average maximum values of $57.6 \pm 1.44,58.5 \pm 2.51$, and $55.8 \pm 1.08$ Gy (RBE), respectively. The mean dose to the brain was significantly lower for both the PSPT and IMPT plans compared to VMAT with average mean values of $7.9 \pm 1.1,10.3 \pm 4.0$, and $17.5 \pm 5.6 \mathrm{~Gy}(\mathrm{RBE})$, respectively. The reduction of the mean dose was expected since the protons have been shown to reduce the volume of surrounding tissue irradiated compared to photon therapies, thereby reducing the mean dose. Kozak et al. [26] also found this reduction in the mean dose to surrounding normal tissues. The increase in the maximum dose is likely due to tissue heterogeneities [28]. Baumert et al. [27] and Kozak et al. [26] also found an increase in the maximum dose from protons compared to photon therapies. Additionally the percentage volume of the brain receiving 5 and 10 Gy (RBE) was significantly reduced for PSPT and IMPT plans compared to VMAT while doses greater than 50 Gy (RBE) showed no significant difference between modalities. VMAT plans resulted in a low dose bath of $10 \mathrm{~Gy}$ (RBE) to nearly 50\% of the brain. PSPT and IMPT reduced the volume receiving $10 \mathrm{~Gy}$ (RBE) to nearly half of that from VMAT. Other studies have found similar results of normal tissue sparing for brain treatments [23,25,27,29-31].

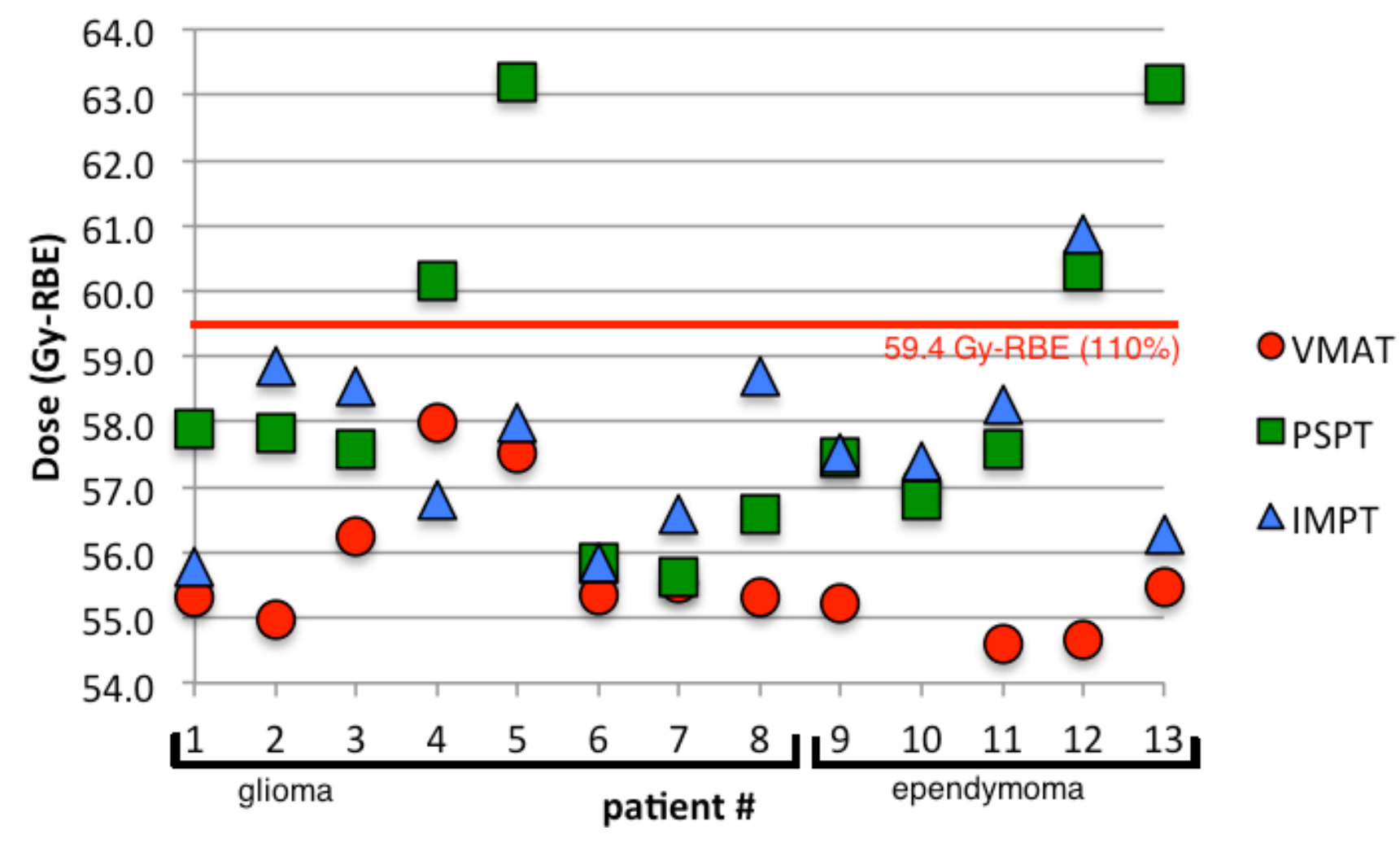

Figure 5. The maximum dose to the brain for VMAT, PSPT, and IMPT plans.

\subsection{Normal Tissue Complication Probability}

The values of rNTCP are displayed in Figure 6, with average values of $0.51 \pm 0.3$ for PSPT compared to VMAT and $0.32 \pm 0.1$ for IMPT compared to VMAT. rNTCP values were compared to 1 using the Wilcoxon signed rank test and were found to be significantly lower than 1, indicating that both proton modalities should confer a reduction in the risk of necrosis. 

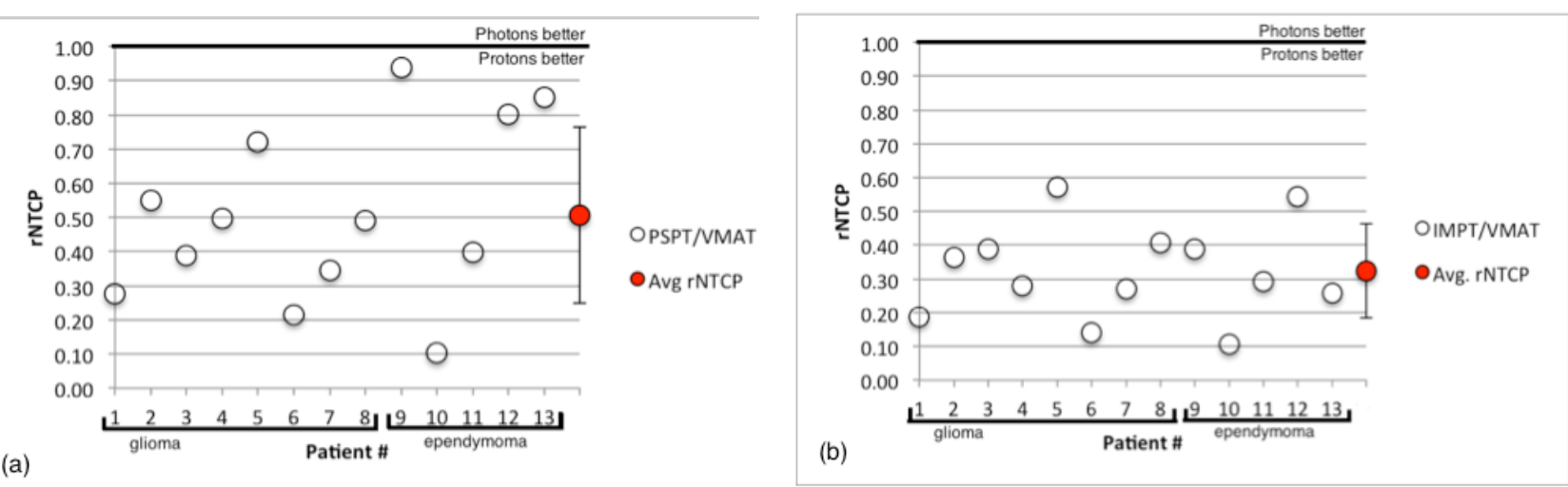

Figure 6. (a) The ratio of risk (rNTCP) for PSPT plans compared to VMAT for all patients. (b) The ratio of risk (rNTCP) for IMPT plans compared to VMAT for all patients. The average value of rNTCP represented by the red circle with standard deviation error bar.

\subsection{Sensitivity Analysis}

Isocenter shifts were performed on patients 5 and 12 . The result of a $1 \mathrm{~cm}$ shift on the ratio of predicted risk can be seen in Figure 7a,c respectively. For patient 5 the greatest change in rNTCP was 0.12 from a right lateral shift in the PSPT plan and 0.18 from a superior shift in the IMPT plan. The greatest changes in rNTCP for patient 12 for PSPT and IMPT plans resulted from a superior shift and left lateral shift and were 0.11 and 0.08 , respectively. Overall the changes in the dose metric for isocenter shifts were minimal and resulted in minor variations in NTCP and the ratio of NTCP compared to the nominal plans. PSPT plans seemed to be slightly more susceptible to setup error, which is likely due to the single beam arrangement that was used in most of the PSPT plans.

Range errors from shifts in the CT calibration curve resulted in changes to the relative risk of necrosis (rNTCP) across proton planning modalities. An increase of $10 \%$ in the calibration curve resulted in reduction of the proton range within the target as well as within the brain and a reduction in the risk. A decrease in the calibration curve would have the opposite effect and an increase in the risk of necrosis was the result. This is in accordance with calibration reports for HU-proton stopping power in the literature [28,32-34]. rNTCP results for calibration curve errors for patients 5 and 12 are displayed in in Figure $7 b, d$, respectively. In both patients an increase in the calibration curve by $10 \%$ resulted in a reduction of rNTCP. A reduction of the calibration curve by $10 \%$ has the opposite effect and increased rNTCP resulting in a decreased benefit for either proton treatments over VMAT. Even with such a large shift all of the proton NTCP values are still less than or equal to VMAT NTCP resulting in a rNTCP of less than 1 for all range errors.

The choice of risk model used can cause large variations in the NTCP results as seen with the alternate risk models chosen in this paper. Most alternative models we considered showed a reduction in the predicted risk when PSPT or IMPT was used instead of VMAT. The ratios of risk from alternative risk model calculations are displayed in Figure 8 for PSPT and IMPT. All models resulted in a ratio of risk significantly less than one with the exception was the PSPT plans calculated using the LT model: The average ratio of risk for these plans was $0.9 \pm 0.2$ and 4 of the 13 plans had a ratio above 1 . The ratio of risk was not significantly less than one for these plans. There was also a single IMPT plan calculated using the LT model that had a ratio of risk above 1, but the average ratio of risk were still found to be 
significantly less than one. Our findings clearly revealed that LKB, LNT, and LP models were strongly influenced by the volume of the brain irradiated at low dose. And although volumes receiving low dose may increase the chance of developing necrosis we believe that necrosis is likely a deterministic effect with a threshold dose and that the high dose region may strongly impact the initial necrotic incident. Additionally, the linear threshold (LT) model had a threshold at about $43 \mathrm{~Gy}$ (RBE) and resulted in absolute risks of necrosis of approximately $1 \%-5 \%$ which better agreed with retrospective studies of similar prescriptions and fractionation and had observed rates of necrosis of approximately $3 \%-5 \%[10,11,14]$.
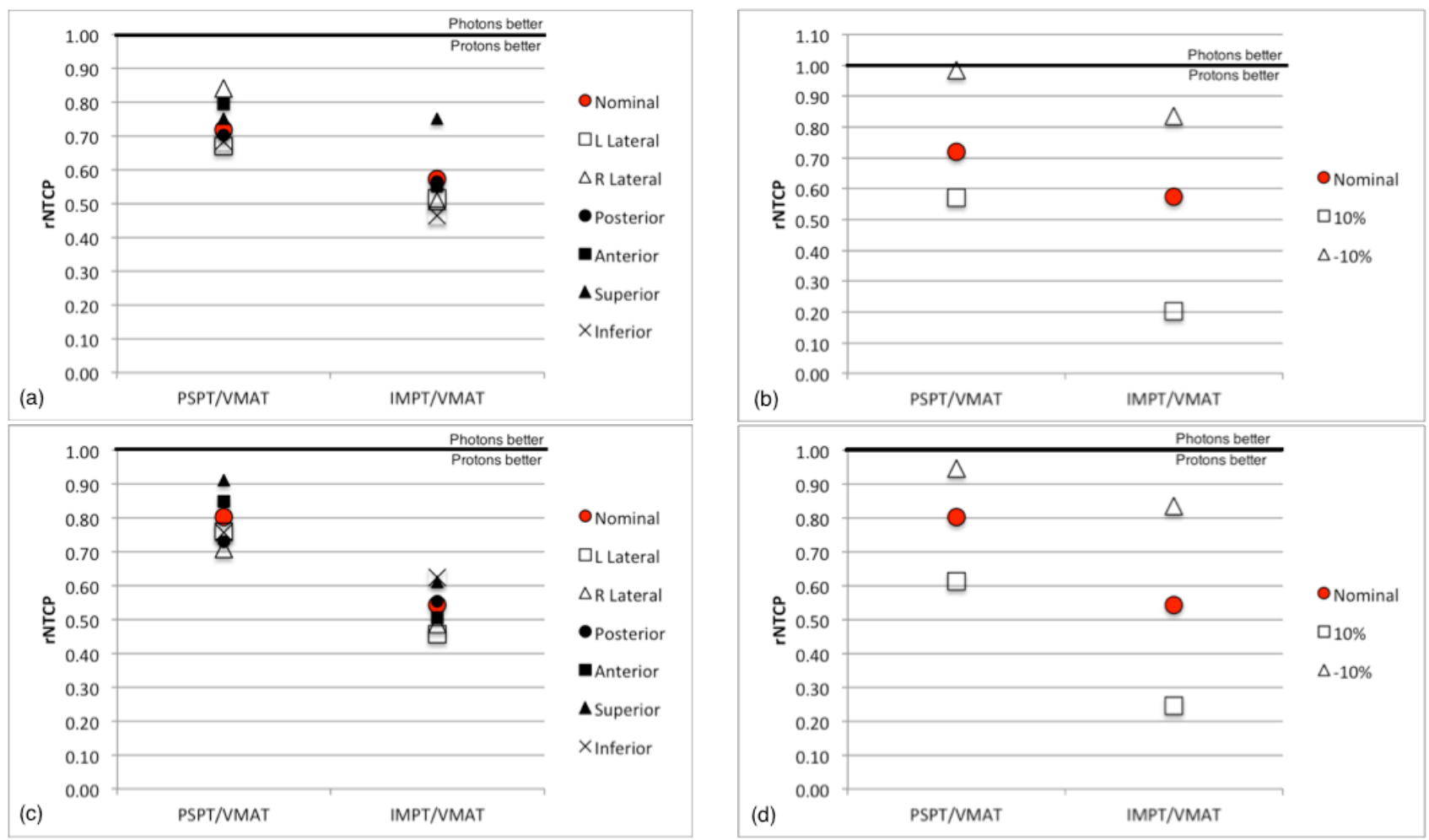

Figure 7. (a) The change in rNTCP with isocenter shifts and (b) with $\pm 10 \%$ calibration curve errors for patient 5. (c) The change in rNTCP with isocenter shifts and (d) with $\pm 10 \%$ calibration curve errors for patient 12 .
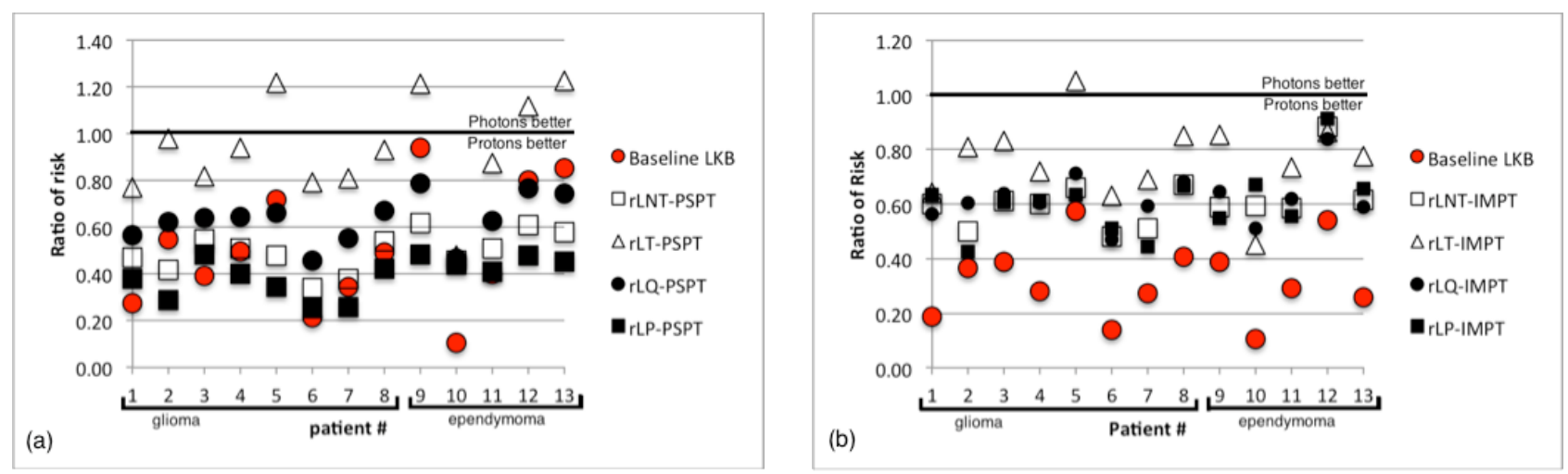

Figure 8. The ratio of risk of necrosis for (a) PSPT plans and (b) IMPT plans compared to VMAT plans, calculated with baseline Lyman Kutcher Burman (LKB) model, the linear no threshold (LNT), linear threshold (LT), linear quadratic (LQ), and linear plateau (LP) models. 


\section{Conclusions}

The risk of radiation necrosis is of particular concern in pediatric patients with brain cancer because the current cure rates for these cancers are high and life expectancy is long and necrosis is a severe side effect of treatment that is potentially fatal. The findings of this study indicate that choosing either PSPT or IMPT over VMAT when treating some pediatric brain cancer patients can reduce the predicted risk of radiation necrosis. The ratio of risk was sensitive to proton range uncertainty but still showed significant risk reduction for proton plans over VMAT. The choice of risk model had little impact on the ratio of risk for necrosis and the qualitative findings of this study still hold in most cases. A more accurate dose-risk model based might be achievable with the availability of a large sample of radiotherapy patient data on necrosis that takes into account dose, volume, fractionation, and RBE variation.

\section{Acknowledgments}

We would like to thank the dosimetry staff at Mary Bird Perkins Cancer Center for their assistance with treatment planning. This work was supported in part by Bella Bowman Foundation, the National Cancer Institute award 1R01CA131463-01A1, and Northern Illinois University through a subcontract of Department of Defense award W81XWH-08-1-0205. The content is solely the responsibility of the authors and does not necessarily represent the official views of the sponsors.

\section{Author Contributions}

Derek Freund performed treatment planning, dose reconstructions, risk calculations and data analysis, and drafted/edited the manuscript. Rui Zhang participated in the design of the project, treatment planning, data analysis and assisted with reviewing/editing the manuscript. Mary Sanders provided guidance on treatment plan design and approved all treatment plans. Wayne Newhauser participated in the design the project, assisted in reviewing/editing the manuscript and provided funding. All authors read and approved the final manuscript.

\section{Conflicts of Interest}

The authors declare no conflict of interest.

\section{References}

1. Dolecek, T.A.; Propp, J.M.; Stroup, N.E.; Kruchko, C. CBTRUS statistical report: Primary brain and central nervous system tumors diagnosed in the United States in 2005-2009. Neuro. Oncol. 2012, 14, v1-v49.

2. Ries, L.; Smith, M.; Gurney, J.; Linet, M.; Tamra, T.; Young, J.; Bunin, G.E. Cancer Incidence and Survival among Children and Adolescents: United States SEER Program 1975-1995; National Cancer Institute: Bethesda, MD, USA, 1999; pp. 35-49.

3. Pizzo, P.A.; Poplack, D.G. Principles and Practice of Pediatric Oncology, 3rd ed.; Lippincott-Raven: Philadelphia, PA, USA, 1997; p. 1522. 
4. Halperin, E.C.; Constine, L.S.; Tarbell, N.J. Pediatric Radiation Oncology; Lippincott Williams \& Wilkins: Philadelphia, PA, USA, 2010.

5. De Laney, T.F.; Kooy, H.M. Proton and Charged Particle Radiotherapy; Wolters Kluwer Health/Lippincott Williams \& Wilkins: Philadelphia, PA, USA, 2008; p. 274.

6. Haas-Kogan, D.A.; Barani, I.J.; Hayden, M.G.; Edwards, M.S.B.; Fisher, P.G. Pediatric central nervous system tumors. In Textbook of Radiation Oncology, 3rd ed.; Phillips, T.L., Hoppe, R., Roach, M., Eds.; Elsevier Publishers: Philadelphia, PA, USA, 2010; p 1111-1129.

7. Ostrom, Q.T.; Gittleman, H.; Farah, P.; Ondracek, A.; Chen, Y.; Wolinsky, Y.; Stroup, N.E.; Kruchko, C.; Barnholtz-Sloan, J.S. CBTRUS statistical report: Primary brain and central nervous system tumors diagnosed in the United States in 2006-2010. Neurooncology 2013, 15, ii1-ii56.

8. Fink, J.; Born, D.; Chamberlain, M.C. Radiation necrosis: Relevance with respect to treatment of primary and secondary brain tumors. Curr. Neurol. Neurosci. Rep. 2012, 12, 276-285.

9. Chao, S.T.; Ahluwalia, M.S.; Barnett, G.H.; Stevens, G.H.; Murphy, E.S.; Stockham, A.L.; Shiue, K.; Suh, J.H. Challenges with the diagnosis and treatment of cerebral radiation necrosis. Int. J. Radiat. Oncol. Biol. Phys. 2013, 87, 449-457.

10. Ruben, J.D.; Dally, M.; Bailey, M.; Smith, R.; McLean, C.A.; Fedele, P. Cerebral radiation necrosis: Incidence, outcomes, and risk factors with emphasis on radiation parameters and chemotherapy. Int. J. Radiat. Oncol. Biol. Phys. 2006, 65, 499-508.

11. Lee, D.-S.; Yu, M.; Jang, H.-S.; Kim, Y.-S.; Choi, B.-O.; Kang, Y.-N.; Lee, Y.-S.; Kim, D.-C.; Hong, Y.-K.; Jeun, S.-S.; et al. Radiation-induced brain injury: Retrospective analysis of twelve pathologically proven cases. Radiat. Oncol. J. 2011, 29, 147-155.

12. Lawrence, Y.R.; Li, X.A.; el Naqa, I.; Hahn, C.A.; Marks, L.B.; Merchant, T.E.; Dicker, A.P. Radiation dose-volume effects in the brain. Int. J. Radiat. Oncol. Biol. Phys. 2010, 76, S20-S27.

13. Lee, A.W.; Foo, W.; Chappell, R.; Fowler, J.F.; Sze, W.M.; Poon, Y.F.; Law, S.C.; Ng, S.H.; O, S.K.; Tung, S.Y.; et al. Effect of time, dose, and fractionation on temporal lobe necrosis following radiotherapy for nasopharyngeal carcinoma. Int. J. Radiat. Oncol. Biol. Phys. 1998, 40, 35-42.

14. Murphy, E.S.; Merchant, T.E.; Wu, S.; Xiong, X.; Lukose, R.; Wright, K.D.; Qaddoumi, I.; Armstrong, G.T.; Broniscer, A.; Gajjar, A. Necrosis after craniospinal irradiation: Results from a prospective series of children with central nervous system embryonal tumors. Int. J. Radiat. Oncol. Biol. Phys. 2012, 83, e655-e660.

15. Boehling, N.S.; Grosshans, D.R.; Bluett, J.B.; Palmer, M.T.; Song, X.; Amos, R.A.; Sahoo, N.; Meyer, J.J.; Mahajan, A.; Woo, S.Y. Dosimetric comparison of three-dimensional conformal proton radiotherapy, intensity-modulated proton therapy, and intensity-modulated radiotherapy for treatment of pediatric craniopharyngiomas. Int. J. Radiat. Oncol. Biol. Phys. 2012, 82, 643-652.

16. Howell, R.; Giebeler, A.; Koontz-Raisig, W.; Mahajan, A.; Etzel, C.; D’Amelio, A.; Homann, K.; Newhauser, W. Comparison of therapeutic dosimetric data from passively scattered proton and photon craniospinal irradiations for medulloblastoma. Radiat. Oncol. 2012, 7, 116.

17. Li, Z. Toward robust proton therapy planning and delivery. Transl. Cancer Res. 2012, 1, 217-226.

18. International Comission on Radiation Units and Measurements. Prescribing, Recording, and Reporting Proton-Beam Therapy ICRU Report 78; Oxford University Press: Oxford, UK, 2007. 
19. Merchant, T.E. Clinical controversies: Proton therapy for pediatric tumors. Semin. Radiat. Oncol. 2013, 23, 97-108.

20. Feuvret, L.; Noel, G.; Mazeron, J.J.; Bey, P. Conformity index: A review. Int. J. Radiat. Oncol. Biol. Phys. 2006, 64, 333-342.

21. Yoon, M.; Park, S.Y.; Shin, D.; Lee, S.B.; Pyo, H.R.; Kim, D.Y.; Cho, K.H. A new homogeneity index based on statistical analysis of the dose-volume histogram. J. Appl. Clin. Med. Phys. 2007, 8, 9-17.

22. Burman, C.; Kutcher, G.J.; Emami, B.; Goitein, M. Fitting of normal tissue tolerance data to an analytic function. Int. J. Radiat. Oncol. Biol. Phys. 1991, 21, 123-135.

23. Merchant, T.E.; Hua, C.-H.; Shukla, H.; Ying, X.; Nill, S.; Oelfke, U. Proton versus photon radiotherapy for common pediatric brain tumors: Comparison of models of dose characteristics and their relationship to cognitive function. Pediatr. Blood Cancer 2008, 51, 110-117.

24. Baumert, B.G.; Lomax, A.J.; Miltchev, V.; Davis, J.B. A comparison of dose distributions of proton and photon beams in stereotactic conformal radiotherapy of brain lesions. Int. J. Radiat. Oncol. Biol. Phys. 2001, 49, 1439-1449.

25. Bolsi, A.; Fogliata, A.; Cozzi, L. Radiotherapy of small intracranial tumours with different advanced techniques using photon and proton beams: A treatment planning study. Radiother. Oncol. 2003, 68, $1-14$.

26. Kozak, K.R.; Adams, J.; Krejcarek, S.J.; Tarbell, N.J.; Yock, T.I. A Dosimetric comparison of proton and intensity-modulated photon radiotherapy for pediatric parameningeal rhabdomyosarcomas. Int. J. Radiat. Oncol. Biol. Phys. 2009, 74, 179-186.

27. Baumert, B.G.; Norton, I.A.; Lomax, A.J.; Davis, J.B. Dose conformation of intensity-modulated stereotactic photon beams, proton beams, and intensity-modulated proton beams for intracranial lesions. Int. J. Radiat. Oncol. Biol. Phys. 2004, 60, 1314-1324.

28. Urie, M.; Goitein, M.; Wagner, M. Compensating for heterogeneities in proton radiation therapy. Phys. Med. Biol. 1984, 29, 553.

29. Fuss, M.; Hug, E.B.; Schaefer, R.A.; Nevinny-Stickel, M.; Miller, D.W.; Slater, J.M.; Slater, J.D. Proton radiation therapy (PRT) for pediatric optic pathway gliomas: Comparison with $3 \mathrm{~d}$ planned conventional photons and a standard photon technique. Int. J. Radiat. Oncol. Biol. Phys. 1999, 45, $1117-1126$.

30. Lin, R.; Hug, E.B.; Schaefer, R.A.; Miller, D.W.; Slater, J.M.; Slater, J.D. Conformal proton radiation therapy of the posterior fossa: A study comparing protons with three-dimensional planned photons in limiting dose to auditory structures. Int. J. Radiat. Oncol. Biol. Phys. 2000, 48, 1219-1226.

31. St Clair, W.H.; Adams, J.A.; Bues, M.; Fullerton, B.C.; La Shell, S.; Kooy, H.M.; Loeffler, J.S.; Tarbell, N.J. Advantage of protons compared to conventional X-ray or IMRT in the treatment of a pediatric patient with medulloblastoma. Int. J. Radiat. Oncol. Biol. Phys. 2004, 58, 727-734.

32. Schneider, U.; Pedroni, E.; Lomax, A. The calibration of CT Hounsfield units for radiotherapy treatment planning. Phys. Med. Biol. 1996, 41, 111.

33. Schaffner, B.; Pedroni, E. The precision of proton range calculations in proton radiotherapy treatment planning: Experimental verification of the relation between CT-HU and proton stopping power. Phys. Med. Biol. 1998, 43, 1579. 
34. Moyers, M.F.; Miller, D.W.; Bush, D.A.; Slater, J.D. Methodologies and tools for proton beam design for lung tumors. Int. J. Radiat. Oncol. Biol. Phys. 2001, 49, 1429-1438.

(C) 2015 by the authors; licensee MDPI, Basel, Switzerland. This article is an open access article distributed under the terms and conditions of the Creative Commons Attribution license (http://creativecommons.org/licenses/by/4.0/). 